\title{
Ciências da atividade física como protagonista de uma agenda multisetorial de pesquisa e advocacy na promoção da mobilidade ativa
}

\author{
Physical activity sciences as protagonist of a multisectoral agenda in the promotion of \\ active transportation
}

\section{AUTORES \\ Ricardo Brandão de Oliveira ${ }^{1,2}$ (iD \\ Rodolfo Guimarães Silva ${ }^{1,2,3}$ (iD \\ 1 Programa de Pós-graduação em Ciências do Exercício e do Esporte Universidade do Estado do Rio de Janeiro, Rio de Janeiro, Rio de Janeiro, Brasil. 2 Laboratório de Vida Ativa, Universidade do Estado do Rio de Janeiro, Rio de Janeiro, Rio de Janeiro, Brasil. \\ 3 Centro Universitário de Volta Redonda, Volta Redonda, Rio de Janeiro, Brasil.}

\section{CONTATO}

Ricardo B. Oliveira

lava.uerj@gmail.com

Rua São Francisco. Rua São Francisco Xavier 524, Maracanã, Rio de Janeiro, Rio de Janeiro, Brasil.

CEP: 20550-900.

DOI

$10.12820 /$ rbafs. $26 \mathrm{e} 0189$

\section{(cc) BY}

Este trabalho está licenciado com uma Licença Creative Commons - Atribuição 4.0 Internacional.

\begin{abstract}
RESUMO
No epicentro da pandemia, as cidades enfrentam desafios econômicos e na saúde. Para lidar com a crise e reduzir os riscos de infecção nos transportes coletivos, o uso de transportes ativos passou a receber atenção de governos locais. Adotando como referencial o conceito de "Saúde em Todas as Políticas", o presente ensaio teórico teve como objetivo discutir oportunidades causadas pela pandemia da COVID-19, considerando as relações entre o uso de transportes ativos e seus impactos sobre a prevenção de doenças crônicas não transmissíveis, a mobilidade urbana e o meio-ambiente. Dentro deste contexto, destacamos a importância de se mensurar as influências e externalidades da indústria automotiva, caracterizando-a como um ator a ser enfrentado nas políticas de promoção da saúde e mobilidade ativa. Neste sentido, acreditamos que as Ciências da Atividade Física podem exercer um protagonismo na formação de uma agenda multisetorial de pesquisa e advocacy, que objetivem reorientar os sistemas de transporte, de desenho urbano e de uso do solo.
\end{abstract}

Palavras-chave: Cidades; Mobilidade urbana; Atividade física e saúde.

\section{ABSTRACT}

At the epicenter of the pandemic, cities face economic and health challenges. In order to deal with the crisis and reduce the risks of infection in public transport, the use of active modes of transportation has received attention from local governments. Adopting the concept of "Health in All Policies" as a reference, this theoretical essay aimed to discuss opportunities caused by the pandemic of COVID-19, considering the relationship between the use of active transportation and its impacts on the prevention of non-communicable diseases, urban mobility and the environment. Within this context, we highlight the importance of measuring the externalities of the automotive industry, characterizing it as an actor to be fought in policies to promote health and active transportation. In this sense, we believe that the Physical Activity Sciences can play a leading role in the formation of a multisectoral agenda for research and advocacy, which aims to reorient the transport, urban design and land use systems.

Keywords: Cities; Urban mobility; Physical activity and health.

\section{Introdução}

No epicentro da pandemia, as cidades são responsáveis por $90 \%$ dos casos diagnosticados da COVID-191. Para muitos centros urbanos, a crise da saúde estende-se como uma crise de acesso a serviços públicos, a alimentação adequada e saudável, a empregos, habitação e transportes, representando, portanto, uma crise de equidade e sustentabilidade que afeta desproporcionalmente as camadas mais vulneráveis da sociedade ${ }^{1}$.

Devido ao fechamento de negócios, postos de trabalhos e demais impactos econômicos, governos locais passaram a ter de lidar com as perdas de suas receitas.
Em reposta a esta crise, líderes políticos deverão potencializar o uso dos recursos através de políticas públicas inovadoras e eficazes, em busca de cidades mais sustentáveis, inclusivas e resilientes.

Durante os meses iniciais da pandemia cidades em diversos países incentivaram a compra de bicicletas e criaram novas ciclovias e zonas para pedestres, recuperando espaços públicos e áreas verdes, melhorando a mobilidade, a segurança e a qualidade do ar. Assim, se por um lado a pandemia impôs desafios à saúde pública, por outro, pode representar uma oportunidade para a adoção de políticas que, na ausência da pandemia, po- 
deriam levar anos para serem implementadas.

Tomando como base o cenário urbano e as relações existentes entre o uso de transportes ativos e seus impactos sobre a saúde, os transportes e o meio ambiente, o presente ensaio teórico faz reflexões sobre as oportunidades consequentes da pandemia da COVID-19, fazendo um chamado para a construção de uma rede de pesquisa e advocacy multisetoriais que pode encontrar nas Ciências da Atividade Física uma importante protagonista. Para esta reflexão, as Ciências da Atividade Física são compreendidas como um campo de investigação das práticas corporais associadas à promoção da saúde, mas também aos sentidos e significados que a elas podem ser atribuídos (sociais, culturais e em diálogo com o ambiente).

\section{Pandemia da COVID-19 - oportunidade para cidades mais saudáveis, sustentáveis e ativas}

Como parte das respostas emergenciais à pandemia, prefeitos de algumas cidades do mundo passaram a recomendar o uso de transportes coletivos apenas para atividades essenciais e, como consequência, espaços públicos destinados aos pedestres e aos ciclistas foram ampliados. Se por um lado observamos a queda das receitas tarifárias de empresas operadoras de transporte, criando uma crise financeira para o setor, por outro, no Brasil, segundo levantamento realizado pela Aliança Bike $^{16}$, a venda de bicicletas em setembro e outubro de 2020 apresentou um aumento de 64\% em comparação ao mesmo período do ano anterior.

Seguindo o exemplo de diversas cidades do mundo, Bogotá $^{17}$, na Colômbia anunciou, em março de 2020, a implementação emergencial de $96 \mathrm{~km}$ de ciclovias temporárias com o objetivo de facilitar o deslocamento diário da sua força de trabalho. Como resultado, o uso das bicicletas cresceu $35 \%$, sendo que $68 \%$ das pessoas que costumavam usar exclusivamente outros meios de transporte passaram a utilizar a bicicleta. De acordo com a prefeita Claudia Lopes, a pandemia da COVID-19 apresenta-se como uma oportunidade única para reduzir o uso de carros, o que trará enormes ganhos políticos, econômicos e ambientais.

No Brasil, cidades como Belo Horizonte, Curitiba e Fortaleza também buscam acelerar a implementação de infraestruturas cicloviárias emergenciais em resposta a pandemia, oferecendo alternativas mais seguras, sustentáveis e saudáveis para os deslocamentos de sua população.

\section{Saúde em todas as políticas - o caso do transporte ativo}

"Saúde em Todas as Políticas" é uma abordagem criada pela Organização Mundial da Saúde que considera os determinantes da saúde e da inequidade em saúde como de origem social, ambiental e econômicos e que, portanto, deve se estender para além do setor e das políticas de saúde.

Trata-se de uma visão que tem por objetivo ampliar o olhar sobre a promoção da saúde através de ações que estão sob controle de setores não ligados tradicionalmente à saúde. Essa abordagem exige uma capacidade aumentada dos sistemas de saúde em engajar outros setores a adotarem políticas que propiciem ganhos em saúde. Isso inclui não apenas melhores habilidades de "advocacy", como também, uma capacidade de identificar relações benéficas que permitam que os demais setores alcancem suas metas ao passo em que se avança na promoção da saúde ${ }^{2}$.

Fruto de uma forte rede multisetorial de advocacy, que conta com o envolvimento da sociedade civil organizada, academia e diferentes setores governamentais, a Convenção Quadro para Controle do Tabaco (CQCT) pode ser considerada um dos mais bem-sucedidos exemplos de "Saúde em Todas as Políticas". Desde sua criação, em 2005, vem implementando a adoção de medidas intersetoriais nas áreas de propaganda, publicidade e patrocínio, advertências, tabagismo passivo, tratamento de fumantes, diversificação, comércio ilegal e elevação de impostos. Reconhecido internacionalmente pela sua liderança no monitoramento, na regulação e no controle do tabagismo, o Brasil reduziu em $31,8 \%$ o número de adultos fumantes entre os anos de 2011 e $2017^{3}$.

Enquanto o transporte ativo segue geralmente negligenciado no planejamento de transportes de muitas cidades, na última década os modais ativos receberam maior atenção nas discussões políticas sobre mobilidade sustentável, mudanças climáticas ${ }^{4}$, pandemia de obesidade e prevenção das Doenças Crônicas Não Transmissíveis (DCNT) $)^{5}$, podendo, atualmente, ser considerado um importante eixo de intervenção de "Saúde em Todas as Políticas".

Os benefícios relacionados ao uso de transportes ativos sobre a saúde são amplos e superam os riscos associados à exposição da poluição do ar e dos acidentes de trânsito ${ }^{6}$. São associados à redução de mortalidade precoce $^{7}$, com impactos equivalentes a políticas públicas consagradas de saúde, como a taxação do tabaco ${ }^{8}$.

Apesar dos avanços no âmbito político com a 
aprovação do Plano Nacional de Mobilidade Urbana (Lei 12.587/2012) e do Programa Bicicleta Brasil (Lei 13.724/2017), que possuem em suas diretrizes a priorização dos modos não motorizados de transportes sobre os motorizados, o que se observa na prática, é o aumento da frota de carros estimulada essencialmente por incentivos fiscais 9 .

Em linhas gerais, os impactos dos transportes motorizados sobre a saúde são: a) acidentes de trânsito, responsáveis por 1,3 milhões de óbitos por ano no mundo $\left.{ }^{10}, b\right)$ maiores níveis de inatividade física e obesidade, c) exposição à poluição do ar, responsável por 1 milhão de mortes por ano no mundo ${ }^{11}$, d) exposição ao barulho das vias, responsáveis distúrbios do sono e hipertensão $\operatorname{arterial}^{12,13}$, e) menor participação social ${ }^{4}, f$ ) mudanças climáticas (carros são responsáveis por 20\% das emissões globais de $\left.\mathrm{CO}_{2}\right)^{4}$.

\section{Promoção da atividade física nos deslocamentos - qual ator devemos combater?}

Em 2011, o Brasil assumiu o compromisso de combater as DCNT com o lançamento do Plano de Ações Estratégicas para o Enfrentamento das Doenças Crônicas não Transmissíveis no Brasil, 2011-2022. Com o objetivo de promover o desenvolvimento e a implementação de políticas públicas efetivas, integradas, sustentáveis e baseadas em evidências para a prevenção e o controle das DCNT e seus fatores de risco, o plano define metas e prioriza diversas ações no campo da alimentação saudável, atividade física, controle do tabaco e uso abusivo do álcool ${ }^{14}$.

Dentre as suas metas, o plano prevê a adoção de medidas que possam garantir a transparência e a eliminação de conflitos de interesses e interferência indevida de indústrias de tabaco, alimentos ultraprocessados e bebidas alcoólicas e açucaradas, por reconhecerem que estas produzem e promovem produtos que causam impactos negativos à saúde, aumento de mortalidade e pobreza ${ }^{14}$. Como resultado, diversos avanços foram observados, como a Lei de Ambientes Livres de $\mathrm{Ci}^{-}$ garro em 2014, através do Decreto no 8.262. Na mesma direção, organizações articulam-se para a regulamentação da rotulagem frontal alimentos, redução de subsídios fiscais à fabricantes de refrigerantes, e proibição de propaganda comercial de bebidas alcoólicas.

Por outro lado, o plano prevê como meta o aumento da prevalência nos níveis de atividade física no tempo livre de lazer da população brasileira em 10\%. Segundo dados do sistema de Vigilância de Fatores de Risco e Proteção para Doenças Crônicas por Inquérito Telefônico (VIGITEL), houve uma tendência crescente para ambos os sexos na prática de atividade física no tempo livre, sendo 14,9\% em 2006 e 20,8\% em 2016, apresentando um aumento anual significativo de $3,6 \%{ }^{15}$. No entanto, diferentemente dos resultados relacionados à prática de atividade física no tempo livre de lazer, quando analisados os seus demais domínios (doméstica, ocupacional e nos deslocamentos), são observadas tendências estacionárias ou decrescentes no período, com destaque para a redução significativa da prática de atividade física nos deslocamentos em 5,9\%, especialmente nas faixas etárias entre 30-39 anos de idade ${ }^{15}$. Cabe destacar que dentre ações intersetoriais propostas no Plano para a promoção da saúde e prevenção de DCNT, são sugeridas articulações com os Ministérios da Educação, do Esporte e da Defesa para a construção do Plano Nacional de Transporte Ativo e Saudável, na perspectiva da segurança pública e do trânsito, da iluminação pública, da mobilidade e da acessibilidade. Sugere-se, ainda, no Plano Diretor das Cidades, a previsão de estruturas que garantam organização e segurança na guarda de equipamentos particulares, como bicicletário, para favorecer o uso de transporte ativo no lazer e no deslocamento para o trabalho ${ }^{14}$.

Isto posto, levantamos alguns questionamentos que consideramos relevantes: a) em um cenário pós-pandemia, pelos motivos já expostos até aqui, não seria urgente a caracterização da indústria automotiva como um ator a ser considerado em políticas públicas associadas à promoção da atividade física e ao combate as DCNT? b) qual seria a responsabilidade da indústria automotiva nas dificuldades encontradas para a implementação das metas do Plano Nacional de Mobilidade Urbana e do Programa Bicicleta Brasil? c) a ausência de um ator claro a ser enfrentado ou regulado podem explicar as dificuldades na formação de redes de advocacy e de uma agenda de pesquisa multisetorial, como as que temos para os demais fatores de risco associados às DCNT? d) existem similaridades entre as ações da indústria do tabaco e automotiva? e) as Ciências da Atividade Física podem exercer um papel de liderança nesse contexto? f) o que podemos aprender com outras redes de saúde?

Responsável por cerca de $15 \%$ do produto interno bruto industrial e um faturamento, em 2015, de 59 bilhões de dólares, o crescimento da produção automotiva segue considerado como um importante impulsionador do crescimento econômico do país, sem que suas 
externalidades sejam devidamente contrabalanceadas por políticas favoráveis à sociedade.

Ao observarmos as ações de lobby da indústria do tabaco e automotiva, notamos muitas similaridades. Tal qual a indústria do tabaco, a indústria automotiva realiza grandes investimentos em marketing para criar a percepção de que seus produtos representam aspiração e status social, construindo, portanto, uma percepção de norma cultural associada a aquisição e uso de carros. Ambas as indústrias possuem empresas associadas que supostamente defendem discursos de regulação como forma de estarem presentes em grupos de influência e decisores políticos que, ao final, atuam para impedir o avanço de leis regulatórias. Muito embora a Taxpayers Alliance, uma organização não governamental europeia, realize campanhas relacionadas ao controle do tabaco, ao aumento de impostos para combustíveis, ao controle de emissão de gases poluentes e a instalação de câmaras de velocidade; sua história possui conflitos de interessantes não declarados, uma vez que parte de seu financiamento é feito por interesse das indústrias do tabaco e automotiva ${ }^{10}$. Por sua vez, enquanto que a FIA Foundation for the Automobile and Society, através da Commission for Global Road Safety, declarou a "Década de Ação para a Segurança das Estradas", segue apenas promovendo medidas reconhecidamente ineficazes como ações educativas voltadas à segurança do pedestre ${ }^{10}$. Cigarro e carros são normalmente posicionados como escolhas e direitos individuais. Ambas as indústrias costumam se utilizar da expressão "Estado Babá" como forma de ataque às ações governamentais que restrinjam o uso de cigarros e reduzam a velocidade dos carros. Esses argumentos enquadram os atos de fumar e dirigir como direitos individuais e que, portanto, não devem sofrer interferências do Estado. Ambas as indústrias financiam pesquisadores e produzem desinformação sobre os efeitos nocivos de seus produtos, como no caso dos dispositivos eletrônicos para fumar. Por fim, ambas as indústrias migram seus mercados para países de baixa e média renda, nos quais as capacidades de governos locais e da sociedade civil organizada em combater suas ações são limitadas. Embora similares, as ações da indústria automotiva são mais difusas e envolvem outros setores produtivos, sendo portanto, de mais difícil caracterização e regulação.

\section{Considerações Finais}

Diante do cenário exposto pela pandemia da COVID-19 e, considerando todo o exposto neste artigo, este ensaio teórico é um chamado para a criação de uma agenda multisetorial de pesquisa e advocacy sobre as influências da indústria automotiva na promoção da mobilidade ativa e as suas consequências na saúde e no enfrentamento das DCNT. Neste sentido, acreditamos que as Ciências da Atividade Física reúnem as condições necessárias para exercer um importante papel de liderança na formação de redes multisetoriais, congregando diferentes campos do conhecimento que permitirão uma maior compreensão dos determinantes sociais e comerciais da mobilidade ativa e a sua relação com o enfrentamento das DCNT.

\section{Conflito de interesse}

Os autores declaram não haver conflito de interesses.

\section{Contribuição dos autores}

Oliveira RB \& Silva RG foram igualmente responsáveis pela concepção, análise e interpretação dos dados, redação do artigo e revisão crítica relevante do conteúdo intelectual, aprovação final da versão a ser publicada e por todos os aspectos do trabalho na garantia da exatidão e integridade de qualquer parte da obra.

\section{Referências}

1. UN. Policy Brief : COVID-19 in an Urban World. United Nations [Internet]. 2020;(July):1-30. Available from: https:// www.un.org/sites/un2.un.org/files/sg_policy_brief_covid_ urban_world_july_2020.pdf.

2. World Health Organization (WHO). Health in all policies (HiAP) framework for country action. Health Promot Int. 2014;29:i19-28.

3. Ministério da Saúde. Secretaria de Vigilância em Saúde. Departamento de Análise em Saúde e Vigilância de Doenças não Transmissíveis. Vigitel Brasil 2018: vigilância de fatores de risco e proteção para doenças crônicas por inquérito telefônico: estimativas sobre frequência e distribuição sociodemográfica de fatores de risco e proteção para doenças crônicas nas capitais dos 26 estados brasileiros e no Distrito Federal em 2018 / Ministério da Saúde, Secretaria de Vigilância em Saúde, Departamento de Análise em Saúde e Vigilância de Doenças não Transmissíveis. - Brasília: Ministério da Saúde, 2019.

4. UNICEF-ONU-UNESCO. Amesterdam declaration - making the link: Transport choices for our health, environment and prosperity. PEP | Transp Heal Environ | Pan-European Program. 2006;71(9):22-3.

5. WHO. Action Plan for the Global Strategy for the Prevention and Control of Noncommunicable Diseases 2008-2013. Geneva World Heal Organ [Internet]. 2008;35. Available from: http://bit.ly/WHO-DCNTs.

6. Mueller N, Rojas-Rueda D, Cole-Hunter T, De Nazelle A, Dons E, Gerike R, et al. Health impact assessment of active transportation: a systematic review. Prev Med (Baltim). 2015;76:103-14.

7. Kelly P, Kahlmeier S, Götschi T, Orsini N, Richards J, Roberts $\mathrm{N}$, et al. Systematic review and meta-analysis of reduction in all-cause mortality from walking and cycling and shape of dose response relationship. Int J Behav Nutr Phys Act. 2014;11(1):132. 
8. Mizdrak A, Blakely T, Cleghorn CL, Cobiac LJ. Potential of active transport to improve health, reduce healthcare costs, and reduce greenhouse gas emissions: A modelling study. PLoS One. 2019;14(7):e0219316.

9. Schapiro MG. O estado pastor e os incentivos tributários no setor automotivo. Brazilian J Polit Econ. 2017;37(2):437-55.

10. Douglas MJ, Watkins SJ, Gorman DR, Higgins M. Are cars the new tobacco? J Public Health (Bangkok). 2011;33(2):160-9.

11. Organization WH. Global health risks: mortality and burden of disease attributable to selected major risks. World Health Organization; 2009. p. vi, 62 p.

12. Stansfeld SA, Matheson MP. Noise pollution: non-auditory effects on health. Br Med Bull. 2003;68(1):243-57.

13. Ising $H$, Dienel $D$, Günther $T$, Markert B. Health effects of traffic noise. Int Arch Occup Environ Health. 1980;47(2):179-90.

14. Ministério da Saúde. Secretaria de Vigilância em Saúde. Departamento de Análise de Situação de Saúde. Plano de ações estratégicas para o enfrentamento das doenças crônicas não transmissíveis (DCNT) no Brasil 2011-2022 / Ministério da Saúde. Secretaria de Vigilância em Saúde. Departamento de Análise de Situação de Saúde. - Brasília : Ministério da Saúde, 2011.
15. Haranaka IP, Soares MMSA, José SN. Tendência dos diferentes domínios da atividade física em adultos brasileiros: dados do Vigitel de 2006-2016. Cad. Saúde Pública. 2020; 36(8):1-12.

16. Aliança Bike. Brasil: setembro e outubro têm vendas de bicicletas $64 \%$ maiores em comparação ao mesmo período de 2019. [Citado em 23 nov 2020]. Disponível em: emhttps:// aliancabike.org.br/vendas-outubro/

17. Bloomberg CityLab. Bogotá Is Building its Future Around Bikes. [Citado em 10 ago 2020]. Disponível em: https:// www.bloomberg.com/news/articles/2020-08-10/to-tametraffic-bogot-bets-big-on-bike-lanes.

Recebido: $31 / 08 / 2020$

Aprovado: 24/02/2021

\section{Como citar este artigo:}

Oliveira RB, Silva RG. Ciências da atividade física como protagonista de uma agenda multisetorial de pesquisa e advocacy na promoção da mobilidade ativa. Rev Bras Ativ Fis Saúde. 2021;26:e0189. DOI: 10.12820/rbafs.26e0189 